\title{
Démonter, extraire, combiner, remonter
}

commodore 64 et créolisation technique

\section{Nicolas Nova}

\section{(2) OpenEdition}

Journals

Édition électronique

URL : https://journals.openedition.org/tc/8478

DOI : $10.4000 /$ tc. 8478

ISSN : 1952-420X

Éditeur

Éditions de l'EHESS

\section{Édition imprimée}

Date de publication : 6 juin 2017

Pagination : 116-133

ISBN : 978-2-7132-2707-3

ISSN : 0248-6016

Référence électronique

Nicolas Nova, « Démonter, extraire, combiner, remonter », Techniques \& Culture [En ligne], 67 | 2017, mis en ligne le 06 juin 2019, consulté le 29 septembre 2022. URL : http://journals.openedition.org/tc/8478 ; DOI : https://doi.org/10.4000/tc.8478 


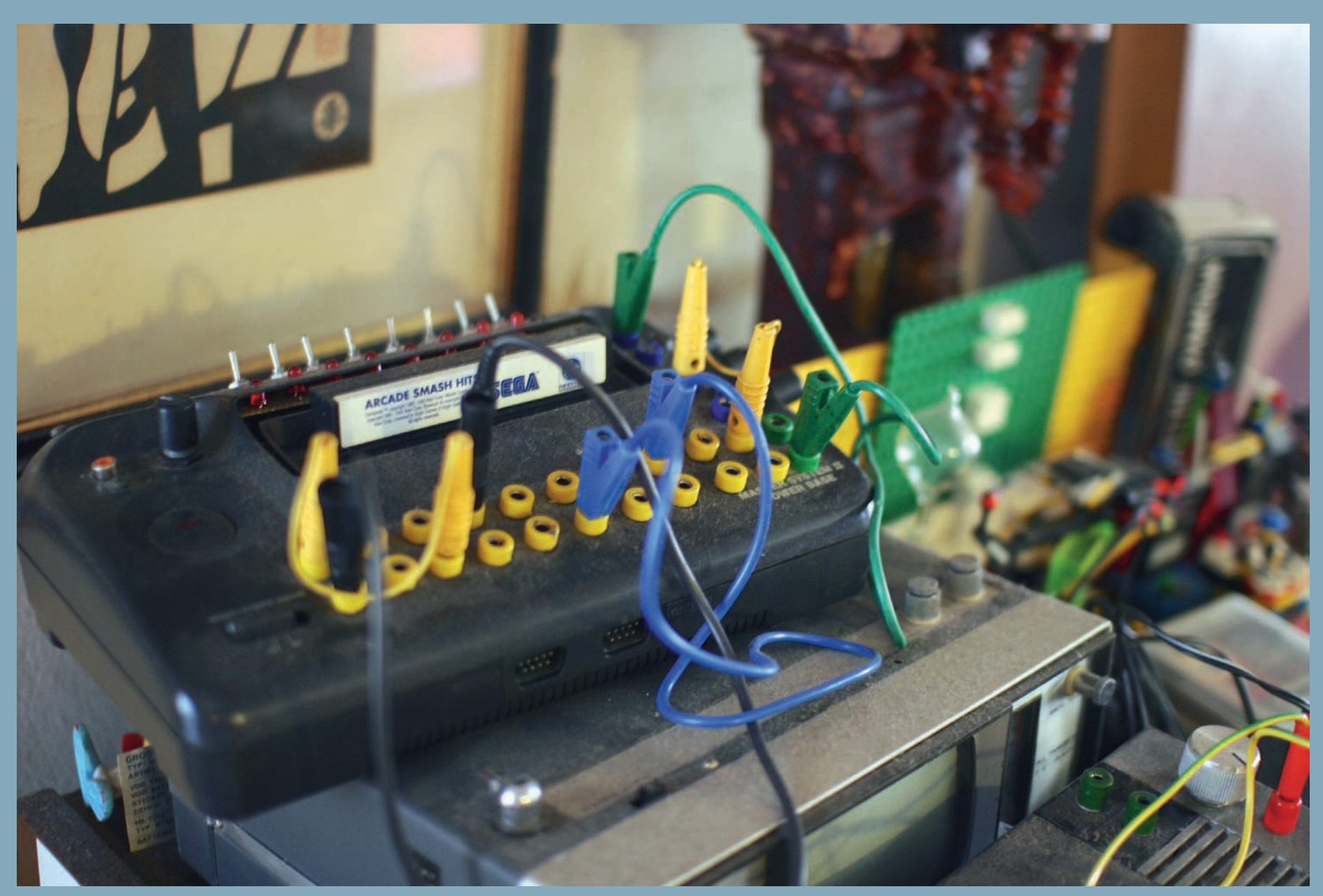




\section{Démonter, extraire, combiner, remonter}

\section{Commodore 64 et créolisation technique}

Dans son ouvrage sur la place de la technique dans l'histoire du xx siècle, l'historien David Edgerton prend le contre-pied du grand récit attribuant un rôle central à l'absolue nouveauté des techniques qui habiteraient les sociétés contemporaines actuelles. Pour appuyer son propos, Edgerton présente une série d'exemples, à partir desquels il propose quelques concepts éclairants. Il décrit en premier lieu, la persistance de techniques prétendues « anciennes », comme c'est le cas dans l'horlogerie de luxe helvétique, ou l'armée de l'Air aux États-Unis qui emploie toujours des avions U-2 des années 1950. Avec la notion de réapparition, de « seconde vie » ou de « technologie rétro », il montre aussi l'importance de la réintroduction au cours du xxe siècle de «vieilles techniques » que furent l'acupuncture, les paquebots ou les répliques de machine à coudre à pédale. L'historien fait également appel à la notion de créolisation pour faire référence à « la diffusion de techniques singulières souvent dérivées de "vieilles techniques" » et renvoyant à des « dérivés locaux de quelque chose originaire d'ailleurs» (Edgerton 2013 : 76). Pour illustrer cette notion, il prend l'exemple de la fabrication d'automobiles au Ghana, ou des taxis « scooterisés » en Inde, en Thaillande et au Bangladesh.

Ces diverses métaphores employées par Edgerton peuvent être considérées comme une déclinaison dans l'espace (introduction dans un autre contexte territorial puis évolution) et éventuellement dans le temps (réintroduction puis évolution) de technologies multiples. Malgré la diversité des cas proposés, et la richesse des descriptions qu'il propose, son ouvrage semble devoir souvent cantonner les «techniques créoles » aux pays en voie de développement, au risque, peut-être parfois, de laisser penser que leur principale caractéristique était de donner « aux techniques originelles importées une seconde vie dans le monde pauvre » (ibid. : 77), que la créolisation n'est qu'un mouvement unidirectionnel des pays riches vers ceux en voie de 
développement. D'où l'intérêt d'interroger et de faire évoluer cette notion de créolisation technique. Pour cela, l'intérêt toujours marqué par les communautés de bricoleurs informatiques et de musiciens pour le C64 - un ordinateur produit par la société canadienne Commodore au milieu des années 1980 -, et en particulier pour son processeur sonore, nous offre un cas d'étude intéressant. Et ce, tout en conservant une leçon importante du travail d'Edgerton qui consiste à ne pas opposer low tech et high tech mais à tâcher de saisir comment des objets techniques « créolisés » relèvent d'hybridations singulières, en creusant à la fois la question de la combinaison, et en tenant compte autant de la dimension temporelle (diachronique) que spatiale des assemblages possibles de technologies dans les dynamiques d'innovation.

\section{Détournement (du) numérique}

Le marché de l'informatique est globalement structuré autour de la production de machines aujourd'hui très standardisées d'ordinateurs personnels, de consoles ou de tablettes commercialisées par les grands industriels internationaux tels que Samsung, Lenovo, Sony, Huawei, Nintendo ou Apple. En parallèle de ce système prédominant, et depuis les débuts mêmes de l'informatique grand public, de multiples communautés de bricoleurs, hackers et autres bidouilleurs se sont attelées à réparer, maintenir, ou modifier les machines datant de différentes époques; des plus récents aux plus anciennes. L'intérêt actuel pour les mouvements makers - cette culture contemporaine du bricolage et de l'apprentissage par la pratique liée à l'idéologie du Do-It-Yourself (« faites-le vous-mêmes ») - et des lieux qui leur correspondent - les hackerspaces $^{1}$ - peuvent être compris comme le prolongement de telles démarches.

Les motivations des participants à ces mouvements sont évidemment diverses, celles-ci reposant soit sur l'intérêt pour la patrimonialisation d'une telle histoire industrielle ${ }^{2}$, soit sur des convictions politiques liées à la réappropriation d’objets techniques fermés (ou pour augmenter la durabilité de ces objets techniques et ainsi en amoindrir les impacts environnementaux ${ }^{3}$ ), soit comme moyen de création. Dans ce dernier cas, par exemple, des artistes ou des créateurs de jeu vidéo revisitent le potentiel graphique ou interactif de technologies parfois plus anciennes. On retrouve aussi cette logique chez les musiciens de la scène dite «Chiptune », qui emploient des machines low tech telles que la Game Boy (Nintendo), l'Amiga (Commodore), ou le C64 (Commodore) disponibles dans les années 1980. Lors d'une enquête antérieure (Nova 2014), je m'étais intéressé à cette micro-scène de musiciens. Mon objectif à l'époque consistait à saisir comment des consoles de jeux vidéo japonaises et des ordinateurs nord-américains avaient été détournés pour produire en particulier du reggae ; et comment deux champs a priori ayant peu à voir l'un avec l'autre - le jeu vidéo et le reggae/dub s'étaient croisés à plusieurs reprises, pour déboucher sur un genre musical underground curieux et intrigant, le « reggae 8-bit ${ }^{4}$. C'est en m’appuyant sur cet exemple que je vais décrire un cas de créolisation technique. Sur la base de mes observations auprès de certains de ces producteurs, et d'une série d'entretiens conduits avec eux, je vais tenter d'éclairer la notion proposée par Edgerton. 


\section{Créer son instrument}

Si la plupart des «musiciens 8-bit » se cantonnent à l'utilisation d'ordinateurs et de consoles, ou à prélever des échantillons sonores sur ces machines - ce qui est déjà intéressant en soi -, d'autres poussent leur passion et plus loin, et créent leurs propres instruments. Le label Jahtari ${ }^{5}$, fondé à Leipzig par Jan Gleichmar (Disrupt) en est un bon exemple. En parallèle de leur activité de production musicale, et sans velléités de production commerciale de masse, il propose aux créateurs de la scène Chiptune le « MIDIbox SID synthesizer », un synthétiseur spécialement dédié à la production musicale ${ }^{6}$. L'appareil, dont ils ont fixé le prix de vente à 1150 euros, consiste en deux blocs de synthèse sonore munis d'une interface de contrôle (boutons, potentiomètres, indicateurs visuels), eux-mêmes insérés dans une coque de Commodore C64 à l'allure caractéristique (un boîtier en plastique gris suivant l'esthétique de l'époque). Chacun des blocs synthétiseurs comprend quant à eux un «SID » (Sound Interface Device) de modèle 6581 ou 8580, le microprocesseur sonore d'un ancien C64. Notons ici que ni cet ordinateur, ni ses composants ne sont encore fabriqués actuellement. Dans les machines de cette période de l'informatique, le rôle de ce composant consistait à générer des sons, et en particulier à reproduire un certain nombre d'instruments.

L'importance du SID pour l'histoire de la musique de jeux vidéo est remarquable puisqu'il fut le premier processeur du genre à être intégré dans un ordinateur grand public, bien avant l'avènement des "cartes sons" des machines actuelles. Son rôle fut également fondamental, du fait de la possibilité de produire plusieurs formes d'ondes sur trois voix, à une époque où les

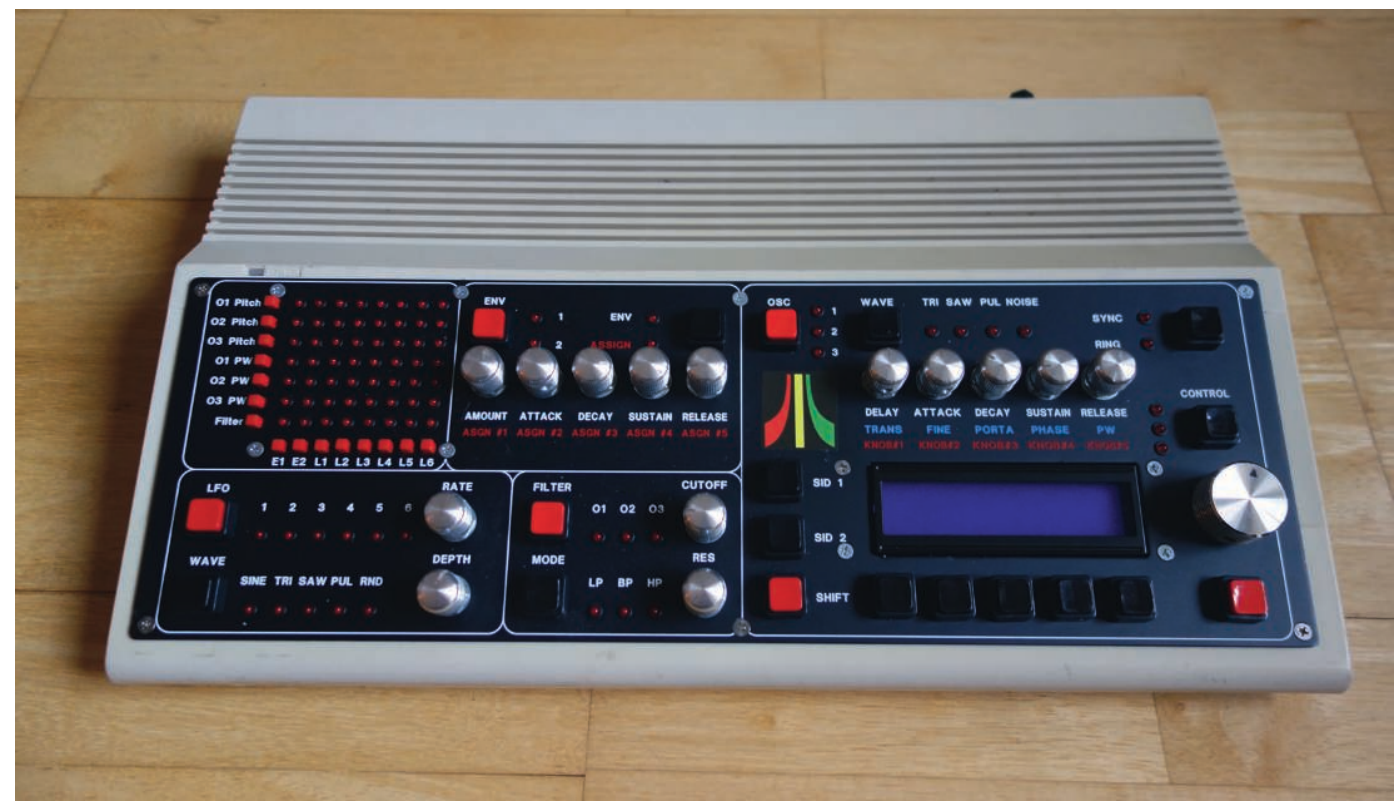

Exemplaire de MIDIbox SID synthesizer produit par Jahtari 


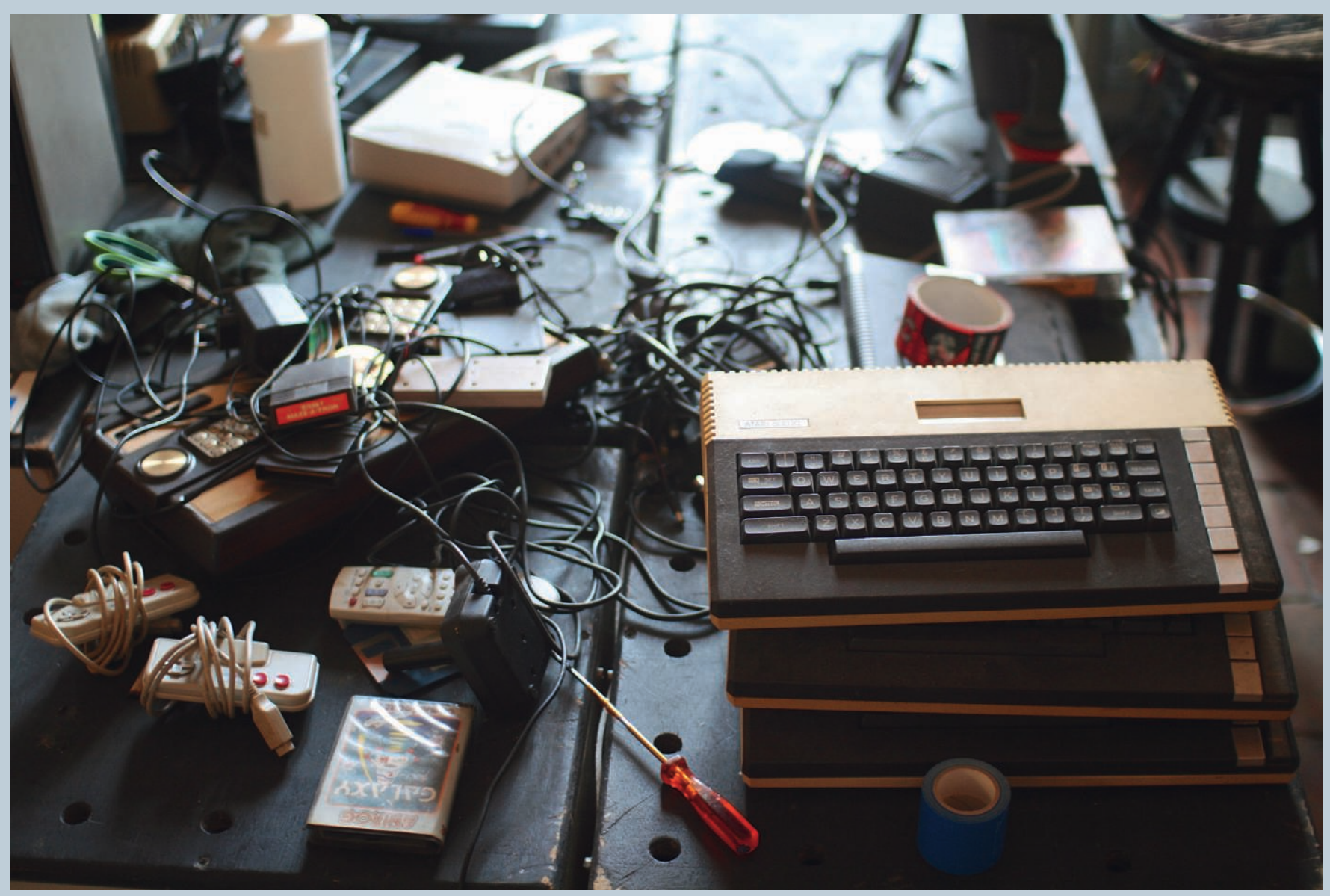

2. Série d'ordinateurs, consoles et périphériques de jeu vidéo à disposition pour être ensuite modifiés ou simplement utilisés dans des festivals de « retrogaming » 
autres ordinateurs n'en étaient pas capables. Rétrospectivement, ce processeur, et plus largement le C64, a eu une importance considérable, documentée par les rares chercheurs à s'intéresser au design sonore et à la musique du jeu vidéo (Collins 2008). En particulier, le type de sons si particuliers et identifiables qu'il permettait de générer (blips and bloops) a marqué les joueurs de l'époque. Leur influence est remarquable, bien au-delà de la sphère du jeu vidéo, comme en atteste la présence de sons-à l'identique dans des morceaux de musique grand public ${ }^{7}$.

\section{Motivations et chaîne opératoire}

Cette démarche qui consiste à prélever des composants « anciens / low tech » pour les combiner à des machines « récentes / high tech » pose deux questions.

Tout d'abord, pourquoi procéder ainsi? Quel est l'intérêt de déployer tant d'efforts à une époque où les échantillonneurs (qui permettent d'enregistrer des extraits sonores manipulables à volonté) sont légion ${ }^{8}$ ? Pour répondre à cette première question, il faut se pencher sur les motivations des musiciens qui acquièrent, ou construisent, de telles machines. Les caractéristiques uniques du SID sont évidemment fondamentales comme le souligne le site de Jahtari :

« Il y a une certaine bizzarerie dans cet objet, et beaucoup de gens qui ont passé du temps avec un C64 (la plupart d'entre eux dans leur jeunesse du fait du jeu vidéo) ont gardé ce son en tête et peuvent directement distinguer un SID d'un autre synthétiseur 9 . » (traduction de l'auteur)

Ce n'est donc pas uniquement une logique de puristes qui veulent produire des sons de la même manière qu'à l'époque (échantillonner les sons suffirait pour cela). Si le processeur sonore était intéressant au sein de l'ordinateur C64, son usage n'était pas forcément très accessible et facile à utiliser avec l'interface (clavier) de cet ordinateur. Prélever le SID et le combiner à des technologies plus récentes est donc une manière de mieux exploiter les possibilités de ce processeur. Comme l'indique le site de Jahtari :

« le problème a toujours été de lui donner un accès direct, pour qu'il puisse être utilisé somme n'importe quel synthétiseur existant (...) Peu importe la quantité de super instruments numériques qu'il y a de nos jours à disposition, il y a toujours le même inconvénient : vous ne pouvez pas vraiment les manipuler à la main. Lorsque vous explorez quelque chose d'aussi complexe qu'un synthétiseur juste avec votre souris, vous vous limitez à la surface de celui-ci. Ce processus qui devrait être très créatif n'est pas très différent d'écrire un e-mail ou de commander certains livres en ligne : clic, clic, clic. Un VRAI synthé c'est autre chose ${ }^{10}$.» $(t d a)$

Le MIDIbox SID synthesizer de Jahtari peut aussi être considéré comme une manière plus pratique de remobiliser ce type de sons - et tout l'imaginaire qui va avec - dans une machine, en en limitant les inconvénients. Pour les musiciens de Jahtari, cette démarche est aussi justifiée par la réappropriation d'un savoir technique et artistique qui a été laissé de côté avec l'avènement 


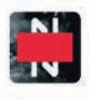

deə|క॥|nN

@Nullsleep

\section{Pulling apart filthy Commodore64s on the kitchen floor at 3am to harvest SID chips, we've all been there before}

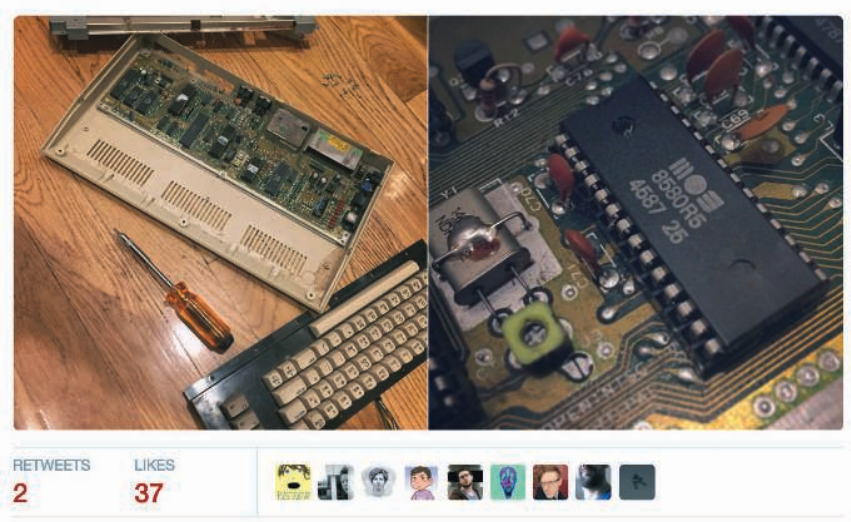

Tweet d'un musicien bricoleur qui témoigne de l'extraction d'un microprocesseur son SID d'un C64, qui sera utilisé dans un autre appareil. Le message atteste aussi du caractère commun d'une telle manœuvre.

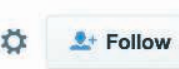

des moyens de contrôle très simples de l'ordinateur que sont la souris et l'interface graphique :

« Lidée derrière cela est de sortir de la zone de confort de l'ordinateur portable et d'apprendre tout sur la création de musique Dub avec de nouveaux outils, et de faire fonctionner les machines d'une manière différente d'un ordinateur. Alors que travailler avec des machines n’a rien de nouveau, il s'agit plutôt de réapprendre ces compétences qui étaient absolument nécessaires jusqu’au début des années 2000, quand un simple ordinateur bon marché pouvait être utilisé comme un instrument. Des compétences qui, d'une façon ou d'une autre, se sont perdues au cours de la dernière décennie, alors que l'on passait du temps à manipuler nos souris. Le plus probable est que le résultat de l'utilisation de la plupart de ces machines pour faire du Dub et du Reggae ne sonnera pas "mieux", mais, j'espère, sonnera un peu différent et plus vivant ${ }^{11}$. »

Comme on le voit dans cet extrait, il s'agit de se réapproprier une technique, et le savoir-faire qui lui est associé, en vue de donner une touche singulière à la musique produite.

Ensuite, pour ces musiciens qui partagent leur temps entre composition en studio (en général dans leur appartement), et les tournées, le fait de ne pas uniquement employer un ordinateur portable sur scène, mais d'utiliser des machines diverses, est un moyen de se distinguer ; surtout lorsque ces appareils ont un cachet aussi fort que celui du C64, et qu'il est personnalisé ou bricolé, sortant ainsi de l'ordinaire. Notons aussi que c'est également un moyen de renouveler cette microscène musicale, - dont aux dires des musiciens et producteursle public s'est parfois lassé -, à l'opposé de concerts présentant des artistes qui manipulent des petites consoles (Game Boy) sur scène. En outre, le fait de « créer ses instruments » correspond à l'émergence, depuis une quinzaine d'années, de toute une communauté d'amateurs bricoleurs intéressés à se réapproprier les technologies numériques, à les modifier et à en expérimenter des usages créatifs. C'est une formalisation du lien mentionné plus haut avec les communautés de makers ou de DIY, dont les acteurs de la scène Chiptune sont une catégorie possible de participants; en particulier ceux s'adonnant au « circuit bending », cette pratique qui consiste à volontairement court-circuiter des instruments de musique électroniques (jouets pour enfants munis de hautparleur, petits synthétiseurs) pour créer des sons originaux.

Une seconde question qu'amène cet objet modifié concerne le « comment », c'est-à-dire quelles sont les différentes opérations matérielles et gestuelles effectuées par Jan Gleichmar et ses collègues pour créer cet appareil. En d'autres termes, pour en comprendre la logique sous-jacente il s'agit de décrire la chaîne opératoire (Leroi-Gourhan 1993, Balfet 1991, Lemonnier 1992) qui mène à ce MIDIbox SID synthesizer. La figure 7, construite sur la base de mes observations, d'entretiens menés avec Jahtari, et la commande que j’ai effectuée, propose une synthèse des différentes étapes. 


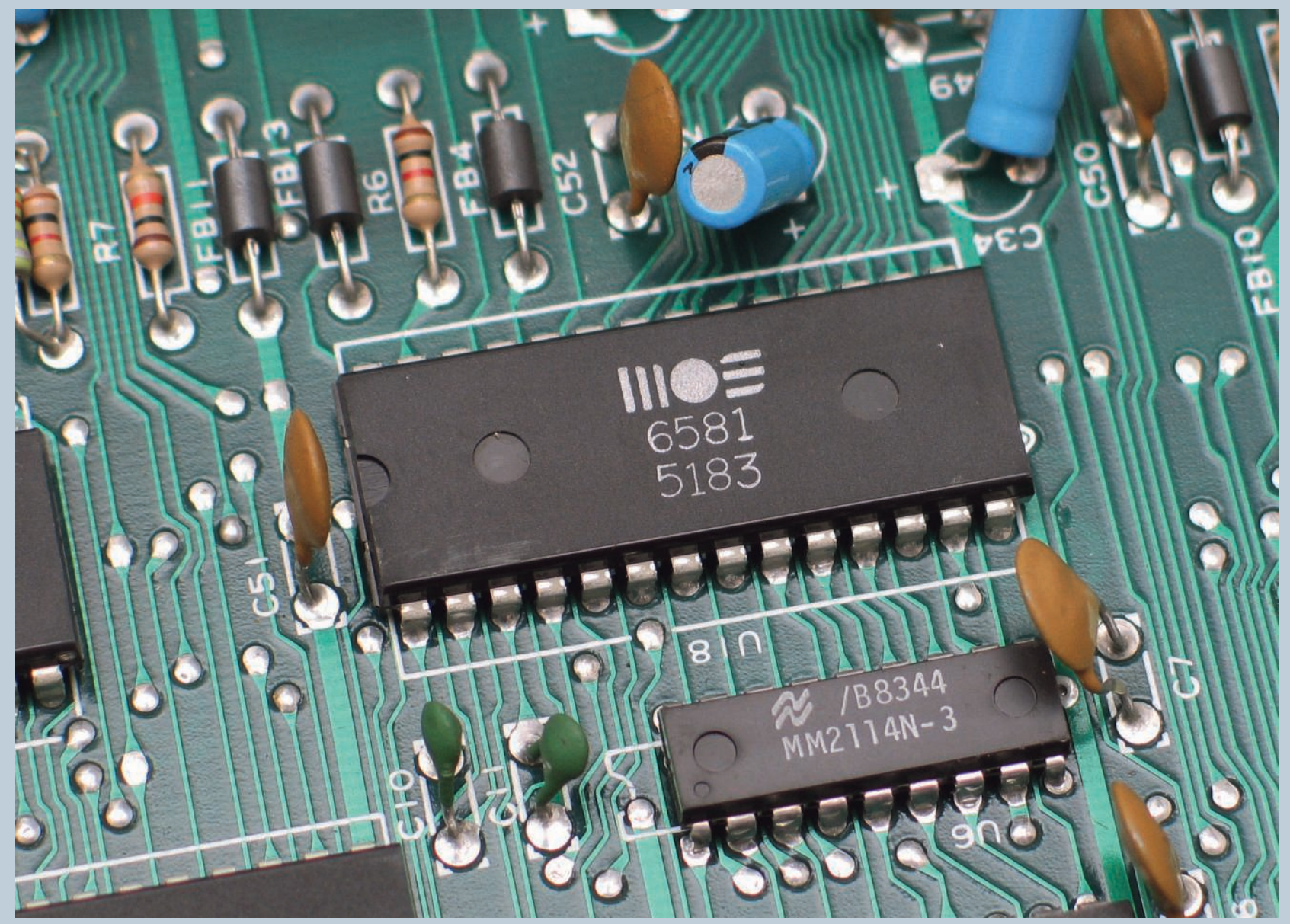

4. Microprocesseur SID (MOS 65815183 ) in situ, sur circuit de C64 Le prélèvement de ce composant consiste à extraire le circuit électronique sans l'abîmer afin de le ressouder dans un autre appareil. 
Site Web du label Jahtari, décrivant le service d'assemblage de différents appareils musicaux, dont le MIDIbox SID synthesizer
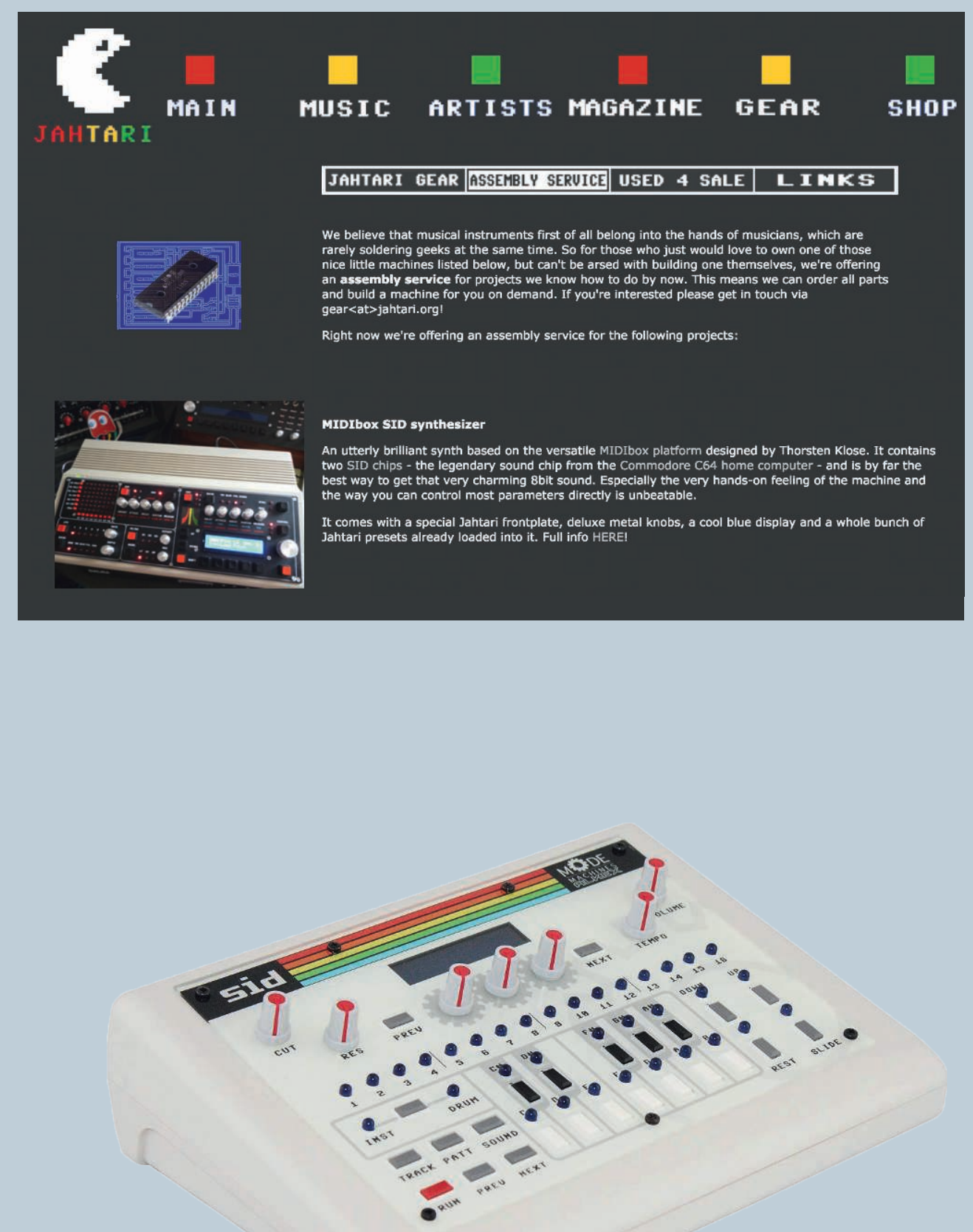

6. SID Groovebox par Mode Machine

Un autre synthétiseur commercialisé, basé sur les sons du C64 


\section{MIDIbox}

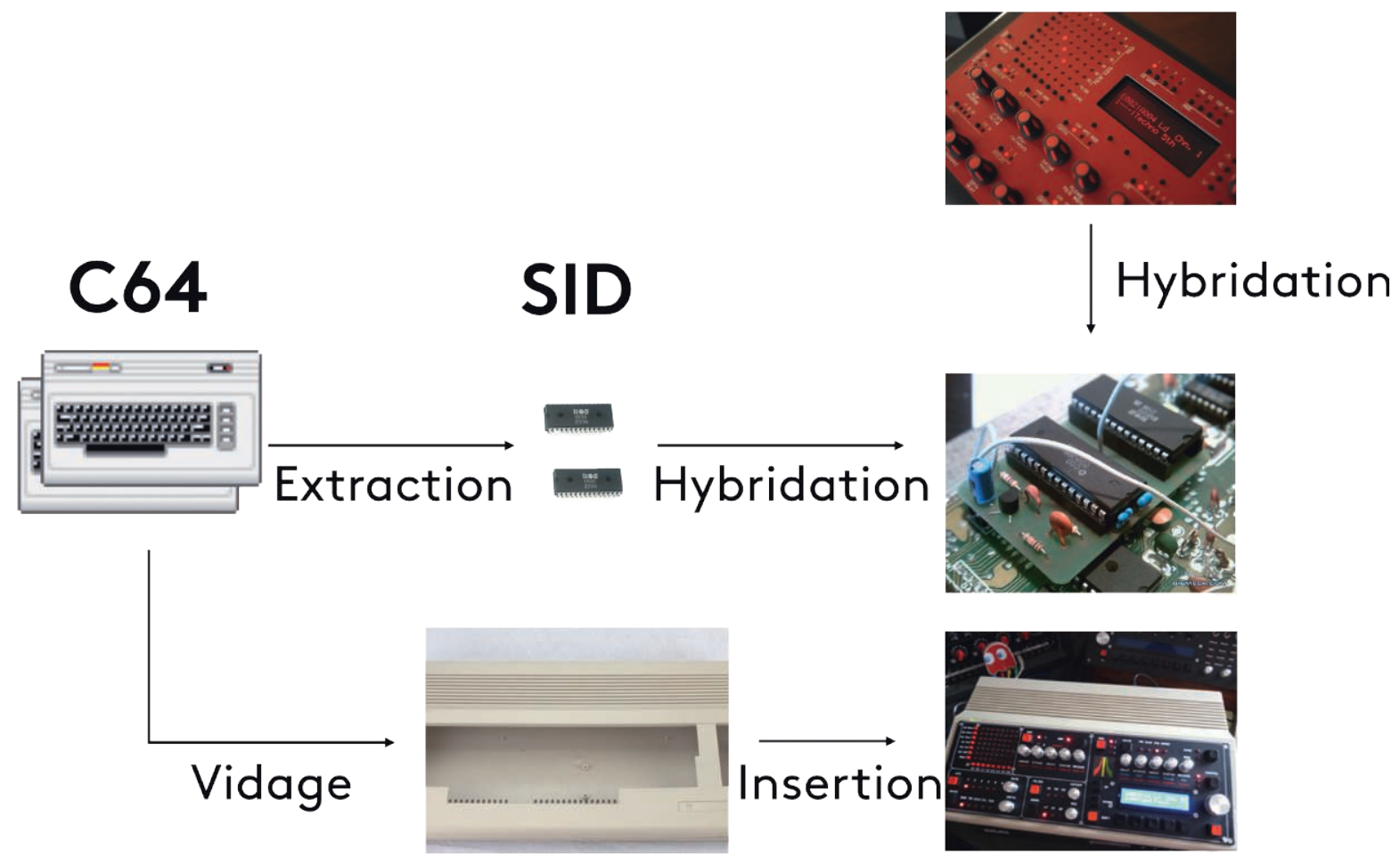




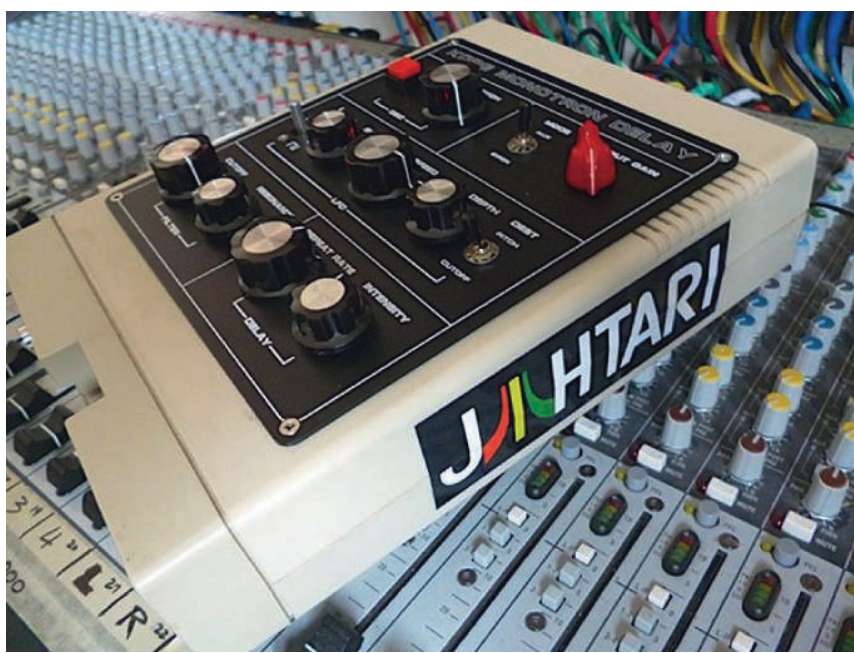

8. Korg Monotron Delay

Étant donné l'absence de productions actuelles des composants SID 6581 et 8580 - et malgré l'existence de copies de mauvaise qualité - la seule source pour les bricoleurs consiste à démonter des ordinateurs C64 pour en extraire les microprocesseurs sonores. En bons passionnés de technologies numériques vintages, ceux-ci possèdent quelques-unes de ces machines en réserve, puisqu'il faut deux de ces machines pour une MIDIbox (celle-ci établie à partir de deux SIDs). Cependant, une partie de leur travail repose sur une surveillance plus ou moins soutenue des plateformes de vente d'objets de seconde main tels que eBay. Pour produire une MIDIbox, Jan Gleichmar (Jahtari) collabore avec Thorsten Klose (uCapps.de) ; lequel se charge de créer le cœur de l'appareil : un double boîtier composé en surface d'un ensemble de boutons de contrôle, deux plaquettes électroniques sur lesquelles sont placés divers composants neufs, et deux microprocesseurs SIDs récemment prélevés sur des C64. Une étape majeure de cette chaîne opératoire consiste à démonter les ordinateurs, et à en prélever le microprocesseur sonore SID. Comme en témoigne la figure 3, qui concerne un autre musicien bricoleur de la scène Chiptune, cette activité semble à la fois ordinaire ("we've all been there before »), et représentative de cette culture des musiciens 8-bit. Les autres composants sont mis de côté pour d'éventuels bricolages expérimentaux, et l'une des coques est conservée afin de servir ensuite comme réceptacle pour y placer le double boîtier nouvellement créé. Ce choix implique éventuellement de percer le plastique pour rajouter des interrupteurs ou des connecteurs audiophoniques. Enfin, comme l'alimentation énergétique peut avoir une influence sur le fonctionnement des SIDs, c'est l'adaptateur électrique du C64 lui-même qui est aussi employé pour faire fonctionner la machine. Une fois finalisé, la coque vissée, et le tableau de contrôle agrémenté d'autocollants au nom du label, l'appareil est, après perception du paiement, envoyé par la poste à son futur.

D'après Jahtari, les musiciens possesseurs d'un tel appareil l'utilisent pour produire toutes sortes de styles musicaux, et très souvent avec d'autres instruments, ou d'autres machines, combinant là encore cet objet low tech/high tech avec d'autres. 


\section{Une créolisation low tech/ high tech}

Démonter, extraire, combiner, remonter. Cette courte description de la chaîne opératoire souligne le non-sens de l'opposition low tech/high tech. La nature des éléments matériels mis en jeu relève de composants anciens (les processeurs sonores, la coque de l'ordinateur réutilisée pour le boîtier final, le bloc d'alimentation électrique), et nouveaux (de nouveaux composants électroniques, d'autres boutons). On se rapproche ici d'une tactique bien connue des ethnographes de la réparation : celle de la « cannibalisation », ce terme employé par les réparateurs, désigne le fait de récupérer les pièces d'un appareil pour en réparer un autre (Callén 2016 : 218) ; sauf qu'ici il ne s'agit pas réparation, mais de création d'un nouvel artefact ayant des fonctions et des moyens de contrôle différents.

À ce propos, pour donner un autre exemple similaire, Jahtari commercialise également des boîtiers d'effets sonores tout à fait récents, de la marque Korg, inclus dans des lecteurs de disquettes de C64 (figure 8). On retrouve ici une logique a minima identique, exception faite de l'étape d'extraction de composants, mais avec la même logique de combinaison (qui n'est ici qu'esthétique).

Tout cela peut ainsi être rapproché de la notion de créolisation technique proposée par Edgerton : « la diffusion de techniques singulières souvent dérivées de "vieilles techniques" [et renvoyant à des] dérivés locaux de quelque chose originaire d'ailleurs » (op. cit. 2013 : 76). Le MIDIbox SID synthesizer, et plus largement la musique Chiptune/8-bit, de par ses sonorités identifiables, renvoyant à une culture bien spécifique, peuvent être compris comme « un dérivé de vieilles techniques », qui elles correspondent à l'usage de processeurs sonores SID ancien (donc low tech). Outre la transposition temporelle, des années 1980 aux années 2010, notons aussi le caractère spatial de ce mouvement. Les bricoleurs de Jahtari étant des « Allemands de l'Est », comme ils se plaisent à le rappeler, qui modifient et combinent des technologies nord-américaines low tech (les SID, le C64) et sud asiatiques (fournisseurs de composants électroniques high tech actuels).

Comme on le voit ici, le cas du MIDIbox SID synthesizer est intéressant en ce qu'il illustre la définition de créolisation technique donnée par Edgerton. Il y a certes une circulation - des composants, de la coque, du bloc d'alimentation -, mais celle-ci s'accompagne d'une combinaison. En outre, le produit de cet assemblage débouche sur de la nouveauté : un rendu visuel différent de l'objet hybridé, des possibilités sonores nouvelles (en partie dues aux limites, incidents et bugs possibles de la machine), un accès facilité aux possibilités des composants SIDs qui sont ici doublés.

Si la notion de créolisation technique est une métaphore intéressante, le cas du MIDIbox SID synthesizer nous montre que ce genre de dynamique sociotechnique peut avoir lieu dans des pays occidentaux jugés « non pauvres »-dans des communautés qui, si elles ne sont pas les plus favorisées économiquement, n'en appartiennent pas moins à la classe moyenne.

Enfin, ce cas de bricolage low tech/high tech peut aussi être lu comme une manière de rouvrir le débat sur la notion de "créolisation ${ }^{12}$. Pour préciser le terme qui, on l'a compris, est un 


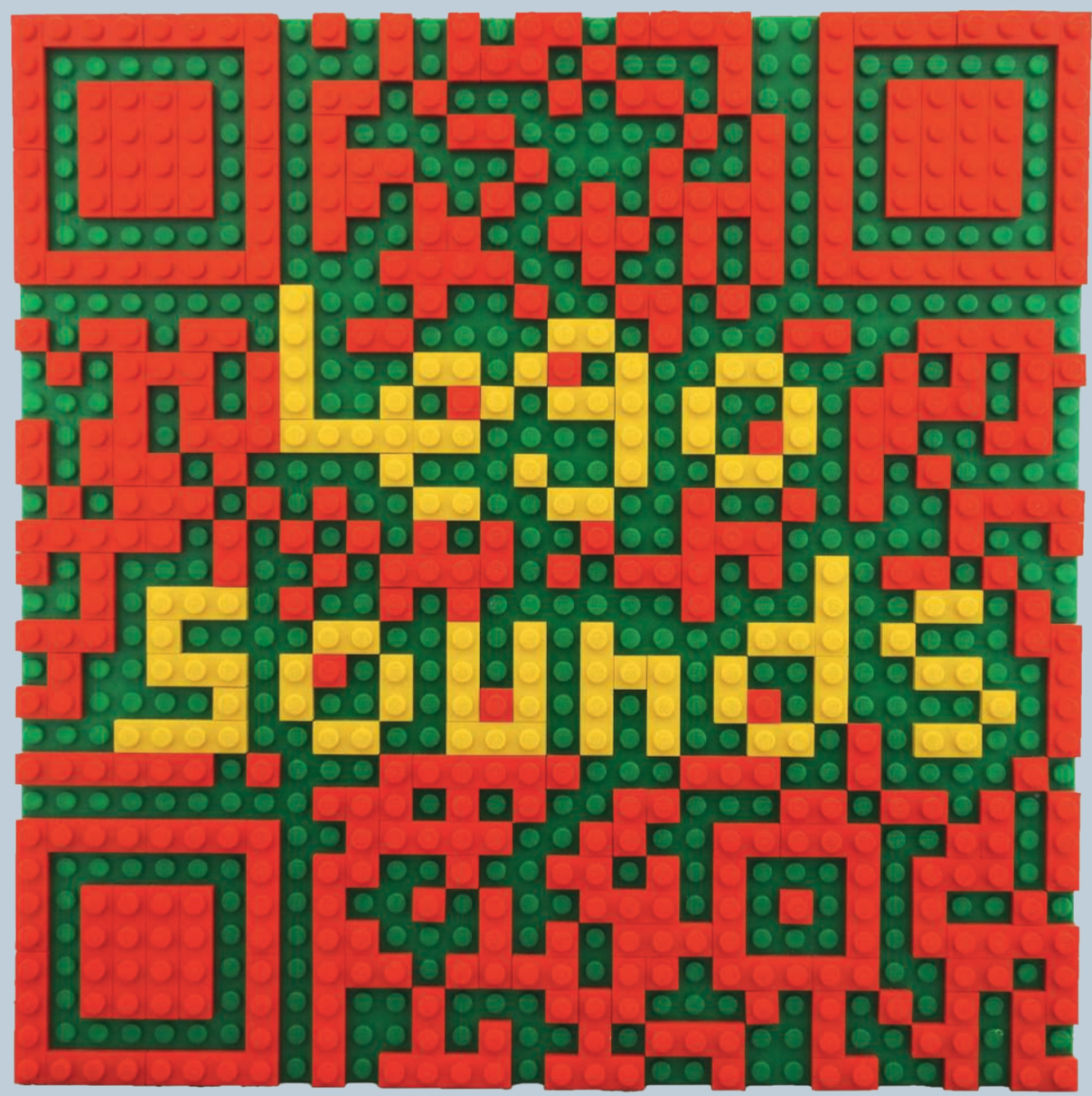

9. Logo du producteur Lego Sounds 


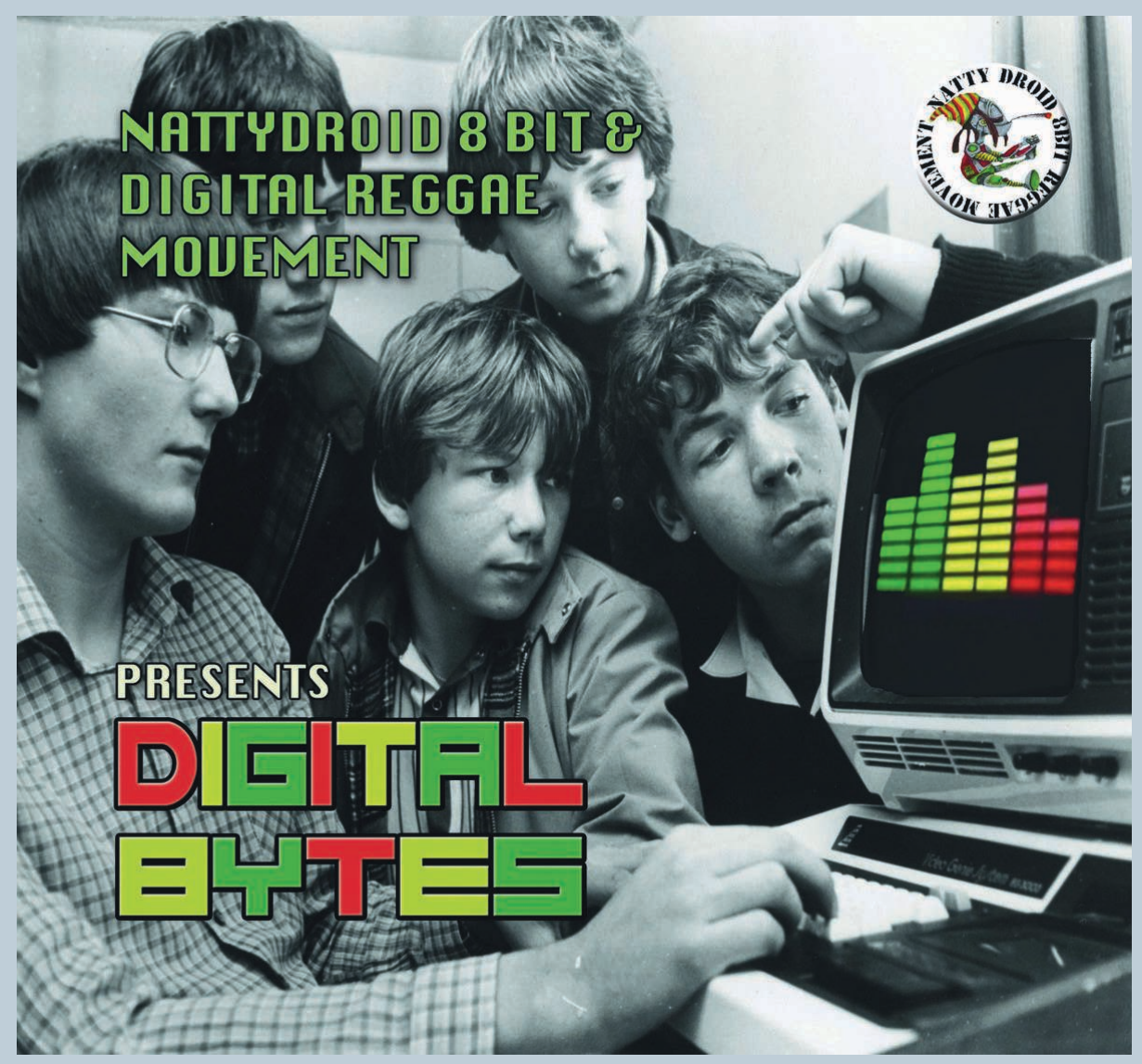

10. Album du groupe Natty Droid 8 bits 
peu vague, également pour sortir d'une terminologie (très connotée) au sens colonial, il peut être intéressant de considérer ces opérations de combinaison d'une autre manière. Le cadre conceptuel de Basile Zimmermann, qui considère les phénomènes de circulation culturelle (Zimmermann 2015) permet d'objectiver l'activité de bricolage de Jahtari. Dans son travail sur le rôle des objets techniques dans la création musicale électronique en Chine, et en observant comment des contenus « occidentaux » peuvent circuler jusque dans les morceaux de musique électronique via les machines, ce sinologue a proposé ce que je nommerai ici une "grammaire de la circulation ». Il s'agit globalement des manières dont la circulation de tout contenu ou objet culturel - les éléments de design d'un objet technique y compris, comme nous l'avons montré dans Zimmermann et Nova (2015) - serait caractérisé par des mouvements de trois ordres : conservation (l'élément reste tel quel), de dissipation (l'élément est éliminé), ou de création (ajout de nouveaux éléments extérieurs). La chaîne opératoire mise en œuvre par les bricoleurs de Jahtari peut donc être comprise comme l'articulation de ces trois logiques, menant in fine à un objet nouveau et singulier, héritant de certaines propriétés des artefacts nécessaires à sa construction : les sonorités 8-bit au cœur de la musique Chiptune. C'est ainsi une autre manière de décrire cette notion de créolisation technique.

\section{Un devenir hybride des objets ?}

Malgré tout l'intérêt que l'on peut porter au MIDIbox SID synthesizer, j'ai bien conscience du caractère très hermétique de ce genre d'objet. Ce n'est évidemment pas un produit grand public et il n'a pas vocation à le devenir. Toutefois, sa description, qui nous a permis de creuser la notion de créolisation technique, est également intéressante puisqu'il existe d'autres biens de consommation qui relèvent de logiques similaires.

Par ailleurs, les étapes suivies par les bricoleurs de Jahtari ne sont finalement pas si éloignées des multiples formes de transpositions de pièces mécaniques ou électroniques recyclées ou réutilisées dans des produits aussi courants que les automobiles (pièces détachées, GPS, autoradio, pneus) ou les téléphones mobiles (changement de batterie, du bloc écran/tactile/bouton, carte SIM) pour ne citer que deux exemples courants. La nuance, par rapport au MIDIbox SID provient ici de l'hybridation low tech/high tech moins courante dans ces champs, et du fait qu'il ne s'agit pas d'un simple transfert puisque les SIDs sont transférés dans un objet différent de celui sur lequel ils ont été prélevés - à la différence d'un transfert de carte SIM d'un téléphone portable à un autre. Dans le champ de l'informatique, les pratiques actuelles d'émulation - qui consistent à substituer un élément de matériel informatique comme un ordinateur ou une console de jeux au moyen d'un logiciel - peuvent aussi être perçues comme une extension de cette logique. Tout comme le succès massif de la commercialisation de vieilles consoles remises au goût du jour, telle la NES Classic Mini ${ }^{13}$ qui est quasiment en rupture de stock depuis sa sortie. De même, ces pratiques rappellent le regain d'intérêt (voire la fétichisation) des appareils photographiques 
vintage ${ }^{14}$. On peut s'en rendre compte tant avec les appareils polaroids « reconditionnés » pour être revendus dans les magasins de la chaîne branchée Urban Outfitters. Comme dans le cas du SID synthesizer, il s'agit de reprendre des composants « anciens » pour concevoir un produit ensuite commercialisé, à la différence d'un autre appareil photo instantané, le LOMO, dont la production de nouveaux modèles n'a pas été interrompue.

Par ailleurs, hormis cette logique d'innovation inhérente à la créolisation, une des conséquences remarquables du cas du MIDIbox SID synthesizer provient du fait que son prix de vente (1150 euros) est bien supérieur à celui des ordinateurs C64 (et des nouveaux composants à rajouter) que l'on trouve actuellement sur le marché de l'occasion (entre 100 et 200 euros le tout). Nous sommes donc en présence d'un objet, qui une fois modifiée et recombinée voit sa valeur augmentée. Tout ceci rappelle en particulier la pratique japonaise du Kintsugi, cette forme d'artisanat qui consiste à réparer de la poterie fêlée en faisant couler un mélange de poudre d'or, d'argent ou de platine dans la fracture... et qui permet de faire revivre l'objet, tout en augmentant grandement sa valeur.

Sans faire de prospective, on peut néanmoins se demander si cette logique d'hybridation intrinsèque à l'appareil de Jahtari ne serait pas un de ces "signaux faibles », annonciateurs soit de pratiques singulières amenées à se généraliser, soit d'une condition nouvelle pour les biens de consommation dans le contexte de la crise écologique actuelle. Nous tenons ici une manière d'innover originale, qui interroge en retour le lien souvent fait entre « innovation » et production de nouveauté... 


\section{Notes}

1. Un hackerspace est un espace communautaire ouvert où les participants peuvent partager ressources et savoir. Les activités, en général liées aux technologies numériques et aux biotechnologies, prennent souvent la forme d'ateliers, présentations et conférences.

2. C'est le cas par exemple de multiples associations qui collectionnent des machines, et qui doivent parfois intervenir sur leur dimension physique ou logicielle afin d'en préserver la viabilité.

3. Comme le montre l'apparition depuis quelques années de lieux de remise à neuf et de maintenance dans les hackerspaces et autres « repair cafés ».

4. Le terme «8-bit » fait référence ici aux caractéristiques techniques des processeurs sonores des consoles de jeu et ordinateurs employés par ces artistes (C64, Game Boy, Amiga).

5. Un mot-valise formé des termes « Jah » (Dieu en Jamaïcain) et « Atari », célèbre marque informatique des années 1970-1990, et qui souligne ici aussi la logique de combinaison-hybridation omniprésente chez ces musiciens/producteurs. Jahtari est un label de reggae et de musique électronique fondé à Leipzig en 2004, qui diffuse sa production majoritairement en ligne.

6. Notons cependant qu'il existe d'autres collectifs de bricoleurs qui crééent des machines similaires, reposant sur la même chaîne opératoire de conception, ou employant un émulateur. C'est le cas par exemple de Mode Machines.

7. Par exemple dans les productions de Snoop Dogg (« Here comes the King ») ou Timbaland (« Tom Ford $»$ ).

8. Une manière courante de procéder chez les musiciens Chiptune consiste à enregistrer les différents sons générés par ce type de processeur sonore, pour ensuite pouvoir les combiner, les assembler tout en les modifiant depuis un programme directement sur l'ordinateur.

9. http://jahtari.org/about/ (traduction de l'auteur)

10. http://jahtari.org/about/ (tda)

11. http://jahtari.org/about/ (tda)

12. Voir notamment l'entrée «Créolisation » dans le dictionnaire des concepts nomades, qui résume la controverse à son sujet (Christin 2016 : 273-280).

13. À l'automne 2016, la société japonaise Nintendo a commercialisé une version modifiée de sa console NES sous la forme d'un appareil de taille réduite, avec une manette de jeu différente et une connectique plus contemporaine (HDMI).

14. Voir à ce sujet Bartholeyns 2015 à propos des enjeux esthétiques et émotionnels sur la photographie rétro.

\section{I'auteur}

Nicolas Nova est professeur à la Haute École d'Art et de Design (HEAD - Genève) et co-fondateur du Near Future Laboratory, une agence de prospective. Auteur de « Beyond Design Ethnography » (SHS Publishing) et « Futurs? La panne des imaginaires technologiques » (Les Moutons Électriques), il enseigne l'ethnographie, les enjeux contemporains du numérique et la recherche en design. Son parcours hybride entre sciences sociales et arts appliqués dans le champ du numérique l'amène à travailler sur des projets de recherche au croisement des démarches ethnographiques et de design.

\section{Iconographie}

Image d'ouverture. Console de jeu vidéo, Master System II (SEGA) modifiée pour des expérimentations Chiptune de « circuit bending » (cf. supra).

Crédits photographiques pour l'ensemble des images

6. Mode Machine

9. Lego Sounds.

10. Natty Droid.

(C) Nicolas Nova sauf:

4. (C) MOS.

$5 \& 8$. Jahtari. 


\section{Références}

Balfet, H. 1991 Observer l'action technique. Des chaînes opératoires, pour quoi faire? Paris : Éditions du CNRS.

Bartholeyns, G. 2015 « Rien ne se perd, rien ne se crée, tout se regrette. Photographies rétro et colorisation », Terrain 65 : 12-33.

Callén, B. 2016 « Donner une seconde vie aux déchets électroniques », TechniquesE Culture 65-66 « Réparer le monde. Excès, reste et innovation » : 206-219. DOI : 10.4000/tc. 7962.

Christin, O. 2016 Dictionnaire des concepts nomades en sciences humaines, tome II. Paris : Métailié.

Collins, K. 2008 « In the Loop : Creativity and Constraint in 8-bit Video Game Audio », Twentieth-century music 4 (2) : 209-227.

Edgerton, D. 2013 Quoi de neuf ? Du rôle des techniques dans l'histoire globale. Paris: Seuil.

— 2007 «Creole Technologies and Global Histories : Rethinking How Things Travel in Space and Time »,
HOST : Journal of the History of Science and Technology $1(1): 75-112$

Lemonnier, P. 1992 « From field to files : description and analysis of technical phenomena ». In P. Lemonnier dir. Elements for an Anthropology of Technology. Ann Arbor: University of Michigan Press.

Leroi-Gourhan, A. 1989 Le geste et la parole. Technique et langage. Paris : Albin Michel.

Nova, N. 2014 8-Bit Reggae: Collision and Creolization. Paris : Éditions Volumiques.

Zimmermann, B. 2015 Waves and Forms : Electronic Music Devices and Computer Encodings in China. Cambridge: MIT Press.

Zimmermann, B. \& N. Nova 2015 «Circulation : A Theoretical Toolkit». Design and Culture 7 (2): 167-184.

\section{Remerciements}

Lauteur remercie Basile Zimmermann, François Ribac, Anaïs Bloch et Pierre-Olivier Dittmar pour leurs remarques et les discussions qu'ils ont suscitées au long de l'élaboration de cet article.

\section{Pour citer l'article}

Nova, N. 2017 « Démonter, extraire, combiner, remonter. Commodore 64 et créolisation technique », TechniquesE Culture 67 « Low tech? Wild tech! », p. 116-133. 